\title{
Fourth International Workshop on Sharing and Reusing Architectural Knowledge (SHARK 2009)
}

\author{
Patricia Lago \\ VU University Amsterdam, \\ The Netherlands \\ patricia@cs.vu.nl
}

\author{
Paris Avgeriou \\ University of Groningen, \\ The Netherlands \\ paris@cs.rug.nl
}

\author{
Philippe Kruchten \\ University of British \\ Columbia, Canada \\ pbk@ece.ubc.ca
}

\begin{abstract}
Architectural knowledge has been recognized by the software architecture community as a self-contained research area in software architecture, and brought along some promising research directions. In this workshop we discuss the issues that lead to the application of architectural knowledge in research and industrial practice, ongoing research and new ideas to advance the field. In its previous editions this workshop examined the state of the art and practice, future challenges and trends, and architectural knowledge as perceived by different research communities, including requirements engineering, service-oriented computing and international standardization. This fourth edition will discuss, among others, the application, experimentation, specialization and use of architectural knowledge theory and approaches.
\end{abstract}

\section{Theme and goals}

The Architecture Knowledge (AK) community is comprised of both researchers and industrial practitioners that are involved in a wide variety of fields, disciplines and application domains [1]. The SHARK workshop is a meeting place for this community, which has grown and matured over the last four years. In its past editions, the workshop explored the state of the art and practice in the field [2], a research agenda driving future $\mathrm{R} \& \mathrm{D}[3]$, and emerging approaches of AK in a broader context [4].

This year the focus is on the application, experimentation, specialization and use of AK theory and approaches. The workshop aims to bring together researchers and practitioners of both the software architecture field, and other communities working on related fields, to provide a more interdisciplinary and multi-faceted perspective on the definition of $\mathrm{AK}$ and on the mechanisms to use it.

\section{Accepted Papers}

We selected 8 papers to be presented in the workshop [5] that we believe will help stimulate discussion and further research.

Perovich et al. present a model-driven approach to designing software architectures organized around architecture design decisions. The design decisions and rationale are encoded in terms of model transformations. The approach is presented for the Enterprise Applications domain and is illustrated with a small example.

Ostacchini \& Wermelinger report on their experience in applying a lightweight approach to assumption management for an Agile software development project. Lessons learned are described along with the areas that require further research.

Farenhorst \& van Vliet report on four years of case study research into architecture knowledge sharing in a large software development organization. The experience gained is presented in terms of four lessons learned and a theoretical framework of activities, which software architects should add to their architecting practice.

Gu \& van Vliet present a study in the area of service oriented architectures. Their goal is to discover the necessary domain-specific architectural knowledge for aiding the architecture decision making process in SOA. The study generalizes from both experience gained in past industrial projects and a study of literature in-the-field. The authors distill four aspects prominent in SOA design, and elicit the related 
knowledge entities that should be codified to effectively support SOA decision making.

$B u$ et al. discuss decision-centric architectural design approaches. Nine such methods are analyzed with respect to three main perspectives: AK modeling, architectural design techniques and design rationale capture and use. Their analysis reveals that most approaches assume that architecturally significant requirements are given, which needs to be further investigated. The authors also identify other weaknesses in the approaches and related areas of improvement.

Unphon presents an empirical study of how to employ software architecture beyond the design phase of product line development. The central idea of the approach is to use architecture as a build hierarchy providing guidance to mediate evolution and design throughout the whole software life cycle.

Ul-haq \& Babar address the important topic of AK codification and structuring with the least possible human intervention. They describe a semi-automatic tool for the extraction of $\mathrm{AK}$ chunks from email and documents. A summary of the design considerations and the implementation of the tool, as well as its early utilization is given. The authors also give an outlook on some interesting issues related to tool extensibility.

De Boer \& van Vliet draw on AK management theory to propose QuOnt, an ontology supporting the reuse of quality criteria in the input stage of software product audits. The paper develops the architectural quality criteria in some depth and refers to some initial experimentation in a semi-automated tool support. While the work has been presented from the perspective of product audits and quality criteria, the decision characteristics of quality criteria indicate that the results are applicable to quality attributes, quality criteria and design decisions alike.

\section{References}

[1] Lago, P.; Avgeriou, P.; Capilla, R. \& Kruchten, P. Wishes and Boundaries for a Software Architecture Knowledge Community WICSA '08: Proceedings of the Seventh Working IEEE/IFIP Conference on Software Architecture (WICSA 2008), IEEE Computer Society, 2008, 271-274

[2] Lago, P. \& Avgeriou, P. First workshop on sharing and reusing architectural knowledge SIGSOFT Softw. Eng. Notes, ACM Press, 2006, 31, pp. 32-36.

[3] Avgeriou, P.; Kruchten, P.; Lago, P.; Grisham, P. \& Perry, D. Architectural knowledge and rationale: issues, trends, challenges, SIGSOFT Softw. Eng. Notes, ACM Press, 2007, 32, pp. 41-46

[4] Avgeriou, P.; Lago, P.; Kruchten, P. Towards Using Architectural Knowledge, SIGSOFT Softw. Eng. Notes, ACM Press, 2009, 34, 4 pp.

[5] SHARK web site: www.cs.rug.nl/ paris/SHARK2009 